\title{
Streptococcus suis type II (group R) as a cause of endophthalmitis
}

\author{
B. F. MCLENDON, A. J. BRON, AND C. J. MITCHELL \\ From the Nuffield Laboratory of Ophthalmology, University of Oxford, and the Department of Pathology, \\ Leicester Royal Infirmary
}

SUMMARY A case is reported of a patient with bilateral endophthalmitis, meningitis, sensorineural deafness, labyrinthitis, and septicaemia due to Streptococcus suis type II (group R). The organism is known to produce epidemic meningitis, septicaemia, and purulent arthritis in piglets, but human infection is rare, and no other case reports of ocular infection are known. The organism was sensitive to penicillin at a minimum inhibitory concentration of $0.03 \mathrm{mg} / \mathrm{l}$.

Streptococcus suis type II is a recognised cause of epidemic infection in piglets, causing meningitis, septicaemia, and purulent arthritis (Field et al., 1954; de Moor, 1963; Elliott, 1966). Human infection occurs rarely and is usually in people whose work involves prolonged contact with live or slaughtered pigs (Zanen and Engel, 1975). Case reports of human disease comprising septicaemia and meningitis have appeared from Denmark (Perch et al., 1968; Koldkjaer and Nielsen, 1971), Holland (Zanen and Engel, 1975), and Great Britain (Hickling and Cormack, 1976).

In the case reported here the patient had, in addition to a septicaemia and meningitis, a sensorineural deafness, labyrinthitis, and severe bilateral endophthalmitis. This is the first report of endophthalmitis secondary to Streptococcus suis type II (group R).

\section{Case report}

A 46-year-old Caucasian man who worked as a maintenance engineer at a pork pie factory was well until 18 November 1976, when he complained of malaise and shivering. Over the succeeding 4 days he developed deafness in the right ear and ringing in both ears, unsteadiness of gait, vomiting, sleeplessness, and irritability. The eyes became watery. A headache developed and the malaise increased. Two days before admission to hospital the left vision deteriorated, and 1 day before admission the eyes became sticky. His general practitioner noted a hazy left cornea and a fixed dilated left pupil in addition

Address for reprints: Mr A. J. Bron, Nuffield Laboratory of Ophthalmology, Walton Street, Oxford OX2 6AW to neck stiffness and referred the patient with a diagnosis of either meningitis or encephalitis.

On admission to the Oxford Eye Hospital on 23 October 1976 the patient was obtunded and his complaints were as described above. He made no complaint of ocular pain or discomfort but mentioned some wrist pain. Systemic examination showed dehydration, purulent pharyngitis, tender swollen wrists, unsteadiness, and a temperature of $37 \cdot 6^{\circ} \mathrm{C}$. The patient was lethargic but responsive to questioning. At this time he had no neck stiffness. Bilateral eighth nerve deafness (worse on the right) and labyrinthitis were noted. No skin lesions were identified, though one observer later noted transient petechiae at the elbows.

Ophthalmic examination showed a visual acuity of 6/9 on the right and hand movements on the left without correction. The intraocular pressures were right $36 \mathrm{mmHg}$ and left $38 \mathrm{mmHg}$. There was bilateral diffuse conjunctival and episcleral injection, and conjunctival haemorrhages were present on the left. In addition there was a haemorrhagic papillary reaction in the upper tarsal conjunctiva. A purulent discharge was present in the left conjunctival sac. The right cornea showed diffuse vesicular epithelial oedema (bedewing) with mild stromal oedema and Descemet's folds. A marked cellular response in the anterior chamber resulted in a red reflex with no fundus view. The pupil was small, round, and reacted sluggishly. The left cornea showed diffuse epithelial and stromal oedema of moderate degree with circumferential infiltration in the paralimbal zone for $360^{\circ}$. Stromal folds were observed. The posterior corneal surface had large curdy precipitates. A deep anterior chamber showed a marked 
flare and cells and a $2 \mathrm{~mm}$ hypopyon. The pupil was fixed in mid-dilation, with no red reflex and no view of the fundus.

A diagnosis of bilateral endophthalmitis with secondary glaucoma, left purulent conjunctivitis, and toxic labyrinthitis with sensorineural deafness was made. Subsequent findings confirmed further diagnoses of meningitis and septicaemia.

\section{LABORATORY FINDINGS}

Bilateral nasal swabs gave no growth, and the conjunctiva grew only Staphylococcus albus. Cytology showed a large number of leucocytes in the left conjunctival smear and a few in the right. The turbid cerebrospinal fluid revealed a pleomorphic cellular picture (polymorphs $331 / \mathrm{mm}$, lymphocytes $29 / \mathrm{mm})$ ) and protein $0.8 \mathrm{~g} /$ litre. Gram-positive cocci were observed in pairs and short chains.

A left anterior chamber tap showed similar Gram-positive cocci. Cultures of the cerebrospinal fluid, aqueous, and blood grew a $\beta$-haemolytic streptococcus which gave a weak reaction with Lancefield group D antiserum (after both acid extraction and autoclaving) but was not resistant to bile. An initial identification of the organism was Streptococcus faecalis. However, the organism was examined by the Streptococcus Reference Laboratory, Colindale, and by Dr S. D. Elliott, Cambridge, and was identified as Streptococcus suis type II. The minimum inhibitory concentration of penicillin and ampicillin was $0.03 \mathrm{mg} / \mathrm{l}$. Haematological investigations included, haemaglobin $14.5 \mathrm{~g}$ /decilitre; haematocrit $44.3 \mathrm{~g} / \mathrm{dl}$, white blood count $8.1 \times 10^{9} / 1$ (polymorphs $84 \%$, lymphocytes $14 \%$, monocytes $2 \%$, and erythrocyte sedimentation rate $94 \mathrm{~mm}$ in the first hour.

\section{TREATMENT}

Initial therapy included benzylpenicillin 4 megaunits 4-hourly intravenously, prednisolone $80 \mathrm{mg}$ daily, probenecid $500 \mathrm{mg}$ 8-hourly, and acetozolamide $250 \mathrm{mg}$ 6-hourly. In addition the patient received bilateral topical bacitracin drops (5000 units $/ \mathrm{ml}$ ) 4 times daily, dexamethasone (Maxidex) drops $0.1 \%$ hourly, atropine drops $1 \%$ twice daily, and subconjunctival benzylpenicillin 500000 units, betamethasone (Betnesol) $4 \mathrm{mg}$, and Mydricaine $0 \cdot 3 \mathrm{ml}$. The patient also received benzylpenicillin 20000 units intraathecally as a stat dose.

After 2 days sytemic antibiotic therapy was changed to ampicillin $1 \mathrm{~g}$ 4-hourly, and subconjunctival ampicillin $100 \mathrm{mg}$ was substituted for crystalline penicillin. The patient received subconjunctival ampicillin and betamethasone on alternate days to each eye. Systemic antibiotic therapy continued for 6 weeks.
PROGRESS

The patient developed a pyrexia which slowly returned to normal with the systemic therapy. The right cornea also cleared, and despite the anterior uveitis no vitreous reaction occurred at any time. The left cornea also cleared, but an increasing hypopyon developed after 1 week of therapy despite a resolution of other signs of inflammation. The hypopyon showed a curious tan colour. Ocular pressures returned to normal in the right eye within 24 hours but remained a persistent problem in the left eye, with readings in the mid 20 s.

An intumescent cataract and pupillary block glaucoma developed as a late complication in the left eye. Extensive iridocapsular adhesions necessitated a left extracapsular lens extraction and broad iridectomy. Postoperatively a thick cyclitic membrane was seen behind the lens capsule. A hazy fundus view at this time showed no focal inflammatory lesions. A persistent postoperative flat chamber due to pupillary block resulted in bullous keratopathy and secondary glaucoma in this eye. At the time of writing the right eye remains entirely normal, with uncorrected vision of $6 / 5$ and intraocular pressure of $16 \mathrm{mmHg}$.

The permanent auditory deficits include no vestibular function on either side. A cold caloric test showed no response from either ear. On the right a severe sensorineural deafness thought to be cochlear or retrocochlear has persisted. On the left a 20 to 30 decibel loss is detected. However, the patient shows good speech discrimination. Although not an auditory cripple, he continues to have balancing problems owing to his total loss of vestibular function.

\section{Discussion}

Previous case reports of Streptococcus suis type II infections in man have described septicaemia and meningitis. Only one earlier human case has shown joint involvement (Hickling and Cormack, 1976). Deafness and vestibular disturbance appear to be the commonest permanent sequelae. In one report left and right sided nystagmus occurred (Zanen and Engel, 1975). Previous reports have not mentioned endophthalmitis or conjunctivitis in man. In 1 report of porcine disease the infected pig appeared to have poor vision (Field et al., 1954).

The origin of infection in piglets is probably the nasopharynx. Some human infections are probably associated with wound infection. It is of interest in the case reported here that a purulent pharyngitis was present. Although the possibility exists that the eyes might have been the site of entry via the conjunctival sac, the sequence of events suggests that 
a septicaemia, as shown by shivering and malaise, predated the ocular symptoms. This would be in keeping with the endophthalmitis being metastatic in origin rather than due to exogenous infection (Shammas, 1977). Metastatic endophthalmitis commonly presents painlessly, with the first ocular symptoms being visual. The reason for this remains obscure, and the lack of pain contrasts with the severity of the uveal inflammation. However, other authors (Walker and Fenwick, 1962) have noted this finding. The possibility must also be considered that the conjunctivitis was metastatic, though it is more likely that it arose from direct inoculation of the organism into the conjunctival sac.

The patient worked as a maintenance man in a pork pie factory. One of his assignments was to alter the operation of the various processing machines when a different type of pie was programmed. This involved priming the machines with raw meat, which gave ample opportunity for contact. The history and clinical course, except for the endophthalmitis, are characteristic of reported infections secondary to Struptococcus suis type II (group R).

This organism appears to be an occupational hazard for people working with raw pig meat. Nine of the 10 human cases reported by Zanen and Engel (1975) worked in close extended contact with live or slaughtered pigs. Of these 10 patients who had purulent meningitis with positive cerebrospinal fluid cultures 5 showed positive blood cultures and 5 showed decreased hearing. The case presented here is the first reported with endophthalmitis.

We acknowledge the help of Dr S. D. Elliott, Department of Pathology, University of Cambridge, and Dr W. R. Maxted, Cross Infection Reference Laboratory, Colindale, for identifying Streptococcus suis Type II. Thanks are due to $\mathrm{Mr}$ A. Freeland for the otological assessment.

\section{References}

Elliott, S. D. (1966). Streptococcal infection in young pigs. Journal of Hygiene, 64, 205-212.

Field, H. I., Buntain, D., and Done, J. T. (1954). Studies on piglet mortality 1. Streptococcal meningitis and arthritis. Veterinary Record, 66, 453-455.

Hickling, P., and Cormack, F. C. V. (1976). Meningitis caused by group $\mathrm{R}$ haemolytic streptococci. British Medical Journal, 4, 1299-1300.

Koldkjaer, O., and Nielsen, G. (1972). Haemolytic streptococcus group R infection. British Medical Journal, 3, 765.

Moor, C. E. de (1963). Septicaemic infection in pigs caused by haemolytic streptococci of new Lancefield groups designated R, S, and T. Antonie van Leeuwenhoek, Journal of Microbiology and Serology, 29, 272-280.

Perch, B., Kristjansen, P., and Skadhange, K. (1968). Group R streptococcus pathogenic for man. Acta Pathologica et Microbiologica Scandinavica, 74, 69-76.

Shammas, H. F. (1977). Endogenous E. coli endophthalmitis. Survey of Ophthalmology, 21, 429-435.

Walker, C. B., and Fenwick, P. (1962). Bilateral fulminating endophthalmitis with streptococcal septicaemia. British Journal of Ophthalmology, 46, 281-284.

Zanen, H. C., and Engel, H. W. B. (1975). Porcine streptococcus causing meningitis and septicaemia in man. Lancet, 1, 1286-1288. 